\title{
Parametric Design of Tensile Structures
}

\section{P. Novysedlák}

Taiyo Europe GmbH.

Mühlweg 2, 82054 Sauerlach, Germany

e-mail: p.novysedlak@taiyo-europe.com

\begin{abstract}
In practice, the design of tensile structures is often subjected to constraints. Besides usual aesthetic and structural requirements also other conditions based on local building codes, project specifications or different technical limitations have to be fulfilled. Finding a solution which satisfies all design requirements is therefore an iterative process. Change in the design is achieved by changing design parameters, which in case of tensile structures can be split in two groups, ie. mechanical and geometrical design parameters. While change of the mechanical design parameters, like membrane prestress or cable pretension, is usually easy to perform on any software platform, some difficulties can arise if changes in the geometrical parameters are necessary. Change in the number of boundary conditions, their spatial position or necessity to reshape the supporting structure can lead to complete remodelling of the structure. In an iterative design process is such a procedure unacceptable. A promising solution for these difficulties in practical design appears in parametrically controlled CAD environments. Inclusion of numerical techniques for design and analysis of tensile structures to existing CAD platforms in form of add-on functionality can release the analyst from labour procedures and can support more effective, or even real-time research of the design space. This work aims to present the achievements, possibilities and limitations of parametric design of tensile structures.
\end{abstract}

\section{REFERENCES}

[1] Nouri-Baranger T., "Computational methods for tension-loaded structures", Archives of Computational Methods in Engineering, 11 (2004).

[2] Dieringer F.H., "Numerical Methods for the Design and Analysis of Tensile Structures", Dissertation at Technische Universität München (2014).

[3] Dührsen T.S.D., "Development of a Parametric Membrane Design Software", Master thesis at IMS Institute for Shell and Membrane Structures (2019). 